\title{
Nanobiosensors: Potentiality towards Bioanalysis
}

\section{Ravindra Pratap Singh}

Department of Biotechnology, Indira Gandhi National Tribal University (Central University), Amarkantak, Anuppur, M.P., India

\section{Editorial}

The biosensor is a device detects an analyte of interest, using a biological component with a physicochemical detector. In another way, a sensor integrates a biological element with a physiochemical transducer to generate an electronic signal, which is proportional to a single analyte detected by a detector. The biologically sensitive elements namely receptors, enzymes, antibodies, nucleic acids, organelles, cell, tissue, microorganisms, and cell which can also be created by biological engineering, which interacts either binding or recognizing with the analyte of interest. The transducer or the detector element (e.g., optical, electrochemical, piezoelectric etc.,) transforms the signal resulting from the interaction of the analyte of interest with the biological element into another signal and then it can be more simply measured and quantified with the help of concerned electronics or signal processing display.

The biosensor is a device detects an analyte of interest. In another way, a sensor integrates a biological element signal, which is proportional to a single analyte detected receptors, enzymes, antibodies, nucleic acids, organelles, created by biological engineering, which interacts either transducer or the detector element (e.g., optical, electrochemical, the interaction of the analyte of interest with the biological measured and quantified with the help of concerned electronics.

The first biosensor was developed by Led and Clark in 1962 and gained much attention. Applications of biosensors in medical diagnostics have motivated scientists towards evolution of biosensor technologies as newer tools due to their simplicity in operation, higher sensitivity and ability to perform multiple analyte analysis. Although, biosensor has potential challenges regarding its performance and yield in terms of simplicity and affordability. The application of biosensor in medical diagnostics and industries like pharmaceutical, food, beverages, environmental, and agricultural have proved immense potentiality because of the high demand in the market e.g., blood glucose level monitoring by glucometer. The biosensor has achieved a high level of precision (very low quantity) in measuring disease specific biomarkers not only in in-vitro but also in in-vivo environment for examples glucose, lactate, peroxides, DNA/RNA, and variety of releasing proteins or antibodies in diseases, which are considered to be potential tool to detect disease at its initial stage and begin treatment early. In order to this development, researchers have started innovative strategies to developed ultrasensitive sensing systems to sense the changes in biological environment e.g., surface plasmon resonance (SPR), graphene, nanotubes, nanowires, or nanocantilevers and quantum dots to quantify very low levels of biomolecules.

The first biosensor was developed by Led and Clark in 1962 medical diagnostics have motivated scientists towards evolution in operation, higher sensitivity and ability to perform challenges regarding its performance and yield in terms in medical diagnostics and industries like pharmaceutical, immense potentiality because of the high demand in the market has achieved a high level of precision (very low quantity) but also in in-vivo environment for examples glucose, lactate, antibodies in diseases, which are considered to be potential early. In order to this development, researchers have started systems to sense the changes in biological environment e.g., or nanocantilevers and quantum dots to quantify very low.
Therefore, the evolution of nanobiosensor based bioanalysis has resulted in substantial progress in its analytical performance and biodetection applications. In the research line of nanobiosensors, important milestones have been achieved for the implementation of a sensitive, affordable, hand-held and portable bioanalytical device with an ultrasensitive limit of detection of the same at the pM-fM level for a few numbers of infectious microorganisms directly in patient's biological samples. Still nanobiosensor is able to selectively detect bioanalyte like uric acid (UA), dopamine (DA) and ascorbic acid (AA) at low level concentration for diagnosing early-stage of neurodegenerative diseases [1-11].

Therefore, the evolution of nanobiosensor based bioanalysis and biodetection applications. In the research line of nanobiosensors, of a sensitive, affordable, hand-held and portable bioanalytical same at the pM-fM level for a few numbers of infectious. Still nanobiosensor is able to selectively detect bioanalyte at low level concentration for diagnosing early-stage of nanobiosensors based on an optical detection method have been fabricated using nanoparticles, quantum dots and nanocantilevers. However the most promising nanobiosensors are the electric detection based for example field effect transistor nanosensors (FET), which is capable of identifying the specific biomarker. They are highly specific to their targets and generate a signal in a very short time within a few seconds. The sensing element of a FET nanobiosensor is the semiconductor channel i.e., nanowire of the transistor made up of using nanosized materials such as carbon nanotubes, and silicon nanowires. These nanomaterials have high surface to volume ratio and become extremely sensitive. A specific recognition entity can be used to immobilize the surface of the nanowire/nanotube, as a result the device specifically sensitive only to a specific target, a single stranded DNA capable of recognizing its complementary strand, an antibody that recognize a specific antigen, that specifically interact with another biological molecule [12-14].

Nanobiosensors based on an optical detection method have However the most promising nanobiosensors are the electric (FET), which is capable of identifying the specific biomarker. Signal in a very short time within a few seconds. The sensing i.e., nanowire of the transistor made up of using nanosized. These nanomaterials have high surface to volume ratio and can be used to immobilize the surface of the nanowire/nanotube, specific target, a single stranded DNA capable of recognizing antigen, that specifically interact with another biological.

Therapeutic drug monitoring is prerequisite for the pharmaceutical drugs with either dosage limitations (using pharmacokinetic and

Corresponding author: Ravindra Pratap Singh, Department of Biotechnology, Indira Gandhi National Tribal University (Central University), Amarkantak, Anuppur (M.P.), Pin-484886, India, Tel: 07629-269775; E-mail: rpsnpl69@gmail.com

Received July 19, 2016; Accepted July 23, 2016; Published July 28, 2016

Citation: Singh RP (2016) Nanobiosensors: Potentiality towards Bioanalysis. J Bioanal Biomed 8: e143. doi:10.4172/1948-593X.1000e143

Copyright: ( 2016 Singh RP. This is an open-access article distributed under the terms of the Creative Commons Attribution License, which permits unrestricted use, distribution, and reproduction in any medium, provided the original author and source are credited. 
pharmacodyanamics) or toxicity issues or both while patients undergoing treatment. Recently, for this purpose nanobiosensors are a class of potential bioanalytical techniques competent in the fast detection of administered many commonly therapeutic drugs or new developed drugs. Researchers are trying to synthesize nanobiosensors, which is biocompatible in nature having high signaling potential, simultaneously delivered along with therapeutic delivery devices for in vivo screening and treatment pertaing to health and diseases [15-19].

Therapeutic drug monitoring is prerequisite for the pharmaceutical and pharmacodyanamics) or toxicity issues or both while nanobiosensors are a class of potential bioanalytical techniques therapeutic drugs or new developed drugs. Researchers are in nature having high signaling potential, simultaneously.

Deaths are high due to infectious diseases locally as well as globally with threats of epidemics, pandemics, emerging, re-emerging diseases, and antibiotics resistance pathogen. The biological or biochemical processes in health and diseases focusing new targets for molecular diagnosis and therapeutics are very crucial to develop not only advance detection methodology but also need to update treatment regimen procedure. The electrochemical and optic based biosensors are well established in clinical chemistry care laboratories for routine testing of blood analytes. Immunosensors as a biosensor lack popularity due to their sensitivity for many bioanalytes when compared to the conventional immunoassay methods. Even though, they exhibit high sensitivity and faster testing of biomarker for cardiac and cancer. To overcome faster error free analysis, POC testing requires by using ultrasensitive transducer technology as a nanobiosesnsor. It is directed towards development of nanobiosensing tools for molecular testing at the community health specifically cerebro, cardiac and cancer testing and to be opened new opportunities for utilizing nanobiosensors. Thus we can say that nanobiosensor is the newer generation biosensor utilizing smart nanomaterials and showed high stability, sensitivity, specificity, accuracy, reproducibility and precision. Smart nanomaterial like quantum dots (QDs), graphene, and carbon nanotubes (CNTs) having ultra high surface area and use as an agent for delivery of drugs, and to develop nanobiodevices, reported by several researchers. Microfluidic nanobiosensors are sensing technology, detects the change of mass on the surface or change of dielectric behavior in the presence of biomarkers in smaller concentration (upto fM). It has multiplexing ability with low detection limit useful for point-of-care and regenerative medicine. Lab-on-a-Chip is a tiny device of diagnostic importance which integrates onto a single chip capable of analyzing one or several parameters. Its application in molecular biology, proteomics and cell biology are great achievement, exihibits more sensitivity and real time monitoring of analytes [20-25].

Deaths are high due to infectious diseases locally as well reemerging diseases, and antibiotics resistance pathogen focusing new targets for molecular diagnosis and therapeutics but also need to update treatment regimen procedure. The in clinical chemistry care laboratories for routine testing due to their sensitivity for many bioanalytes when compared exhibit high sensitivity and faster testing of biomarker POC testing requires by using ultrasensitive transducer of nanobiosensing tools for molecular testing at the community and to be opened new opportunities for utilizing nanobiosensors. Biosensor utilizing smart nanomaterials and showed high and precision. Smart nanomaterial like quantum dots (QDs), surface area and use as an agent for delivery of drugs, microfluidic nanobiosensors are sensing technology, detects behavior in the presence of biomarkers in smaller concentration limit useful for point-of-care and regenerative medicine.
Which integrates onto a single chip capable of analyzing biology, proteomics and cell biology are great achievement.

The understanding of human physiology is much more progressing because of emerging fields of genomics, proteomics, mutations and metabolomics. Due to these emerging fields, rational design approaches to diagnosis, drug development, personalized medicine and point-ofcare devices (POCDs) are possible. Personalized medicine and pointof-care testing techniques must provide a cost-effective alternative to expensive and time-consuming laboratory tests, which help to clinicians or health care personnel for the disease diagnosis and treatment/cure regimen. A new type of high-sensitivity and low cost sensors, called plasmonic biosensors or nanobiosensor, could ultimately become a key asset in personalized medicine by helping to diagnose diseases at an early stage. Personalized medicine is a new development for the health care for the detection of biomarkers like nucleic acids, proteins, metabolites in blood or saliva or urine and pathogens. Design and fabrication of ultra-sensitive biosensors for early personalized diagnostics is an urgent need to meet early detection of biomarkers, which indicate the presence of diseases much before the appearance of sign and symptoms, whose presence or abnormal concentration is caused by a disease. Currently the detection of these biomolecules still requires specialized laboratories and is costly. POCDs are widely used in the clinical tests for detection of certain diseases like blood glucose levels, pregnancy tests, etc. Major benefits of POC devices is that analyses which can be done in minutes rather than much longer times, as hours needed in conventional analytical labs [26,27].

The understanding of human physiology is much more progressing and metabolomics. Due to these emerging fields, rational medicine and point-of-care devices (POCDs) are possible. Must provide a costeffective alternative to expensive and or health care personnel for the disease diagnosis and treatment/cure called plasmonic biosensors or nanobiosensor, could ultimately diagnose diseases at an early stage. Personalized medicine of biomarkers like nucleic acids, proteins, metabolites of ultra-sensitive biosensors for early personalized diagnostics which indicate the presence of diseases much before the concentration is caused by a disease. Currently the detection and is costly. POCDs are widely used in the clinical tests pregnancy tests, etc. Major benefits of POC devices is that a biosensor based diagnostic technology can detect a range of clinical analytes of interest quickly. Few examples are portable glucometers for sugar testing, hCG based pregnancy testing kits and malaria testing kit, etc. which are extensively fabricated and possess good market potential. Furthermore research to improvise these existing designs into nanobiosensors which are still in infancy stage. As we know that R \& $\mathrm{D}$ to design nanobiosensor prototype has been rapid and ever expanding due to unique properties nanoscale materials utilization. R\&D in the field of nanobiosensors is the multidisciplinary with diverse domain. A highly sensitive and interference free detection system using nanobiomaterials have been developed as nanobiosensor for the detection of variety of clinically important target molecules including, hormones, cardiac and cancer biomarkers, cancer cells, parasites, bacteria, viruses, heavy metal ions etc., [28].

A biosensor based diagnostic technology can detect range portable glucometers for sugar testing, hCG based pregnancy fabricated and possess good market potential. Furthermore which are still in infancy stage. As we know that $\mathrm{R} \& \mathrm{D}$ expanding due to unique properties nanoscale materials utilization. with diverse domain. A highly sensitive and interference as nanobiosensor for the detection of variety of clinically and cancer biomarkers, cancer cells, parasites, bacteria. Nanobiosensors based on plasmon for the detection of analytes of 
interests have been repoted by several researchers. This achievement, health-care programmes are fastly moving to prevention and early detection of diseases. It will be a component of future medicine and approach will make medical care more cost effective and ecofriendly. So that such type of devices with the unique features based on plasmonics are still needed [29-31].

Smartphones based nanobiosensor is handy, well equipped with advanced processors, increased memory, high-resolution camera, high end security and a variety of in-built sensors. This prototype for in-vitro and real-time monitoring of clinical ananlytes of interest has been reported. Electrochemical biosensing, immunoassays, SPR based biosensing, flow cytometry, and optical detection based smartphone nanobiosensor are under progress and even near towards commercialized which will asset for monitoring and management in critical conditions [32-35].

Nanobiosensors based on plasmon for the detection of analytes achievement, health-care programmes are fastly moving to component of future medicine and approach will make medical of devices with the unique features based on plasmonics.

Thus still nanobiosensor promises continuous R\&D to design not only novel sensing but also enhance the known sensing strategies. The bionanosensor would be directed towards bioanalysis with high sensitivity, selectivity, specificity, quick, with high reproducibility, precision and accuracy, which are still in progress.

Thus still nanobiosensor promises continuous R\&D to design strategies. The bionanosensor would be directed towards quick, with high reproducibility, precision and accuracy.

\section{Acknowledgement}

Dr. Ravindra Pratap Singh (R.P. Singh) thanks to IGNTU, Amarkantak, M.P. India for providing facilities to prepare this editorial.

\section{References}

1. Ravindra PS (2011) Prospects of Nanobiomaterials for Biosensing. International Journal of Electrochemistry, publisher SAGE-Hindawi journal collection p: 30.

2. Ravindra PS (2012) Prospects of Organic Conducting Polymer Modified Electrodes: Enzymosensors. International Journal of Electrochemistry p: 14.

3. Cooper MA (2002) Optical biosensors in drug discovery. Nat Rev Drug Discov 1: $515-528$.

4. D'Orazio P (2011) Biosensors in clinical chemistry2011 update. Clin Chim Acta 412: $1749-1761$

5. Yu DH, Blankert B, Vire JC, Kauffmann JM (2005) Biosensors in drug discovery and drug analysis. Anal Lett 38: 1687-1701.

6. Ravindra PS, Oh BK, Koo KK, Jyoung JY, Jeong S, et al. (2008) Biosensor Arrays for Environmental Pollutants Detection. Biochip Journal 2: 223-234

7. Ravindra PS, Kim YJ, Oh BK, Choi JW (2009) Glutathione-s-transferase based electrochemical biosensor for the detection of captan. Electrochemistry Communications (ELECOM) 11: 181-185.

8. Ravindra PS, Kang DY, Oh BK, Choi JW (2009) Polyaniline based catalase biosensor for the detection of Hydrogen peroxide and Azide. Biotechnology and Bioprocess Engineering 14: 443-449.

9. Arora K, Chaubey A, Singhal R, Singh RP, Pandey MK, et al. (2006) Application of electrochemically prepared polypyrrole-polyvinylsulphonate films to DNA Biosensor. Biosensors and Bioelectronics 21: 1777-1783.

10. Arya SK, Solanki PR, Singh RP, Pandey MK, Datta M, et al. (2006) Application of octadecanethiol self assembled monolayer to cholesterol biosensor based on surface Plasmon Resonance technique. Talanta 69: 918-926.

11. Ravindra PS, Jeong WC (2009) Biosensors development Based on Potentia Target of Conducting Polymers. Sensors \& Transducers Journal Publisher: International Frequency Sensors Association 104: 1-18.
12. Molaie M (2014) Field effect transistor nanobiosensors: State-of-the-art and key challenges as point of care testing devices. Nanomedicine J 3: 69-82.

13. Sagadevan S, Periasamy M (2014) Recent trends in nanobiosensors and their applications-a review. Rev Adv Mater Sci 36: 62-69.

14. Chen KI, Li BR, Chen YT (2011) Silicon nanowire field-effect transistor-based biosensors for biomedical diagnosis and cellular recording investigation. Nano Today 6: 131-154.

15. Carrara S, Cavallini A, Erokhin V, De Micheli G (2011) Multipanel drugs detection in human serum for personalized therapy. Biosens Bioelectron 26 3914-3919.

16. Bossmann SH, Troyer DL (2013) Pointofcare routine rapid screening: the future of cancer diagnosis. Exp. Rev Mol Diagn 13: 107-109.

17. Martinez AW, Phillips ST, Whitesides GM, Carrilho E (2009) Diagnostics for the developing world: microfluidic paperbased analytical devices. Anal Chem 82: 3-10.

18. Singh RP, Choi JW, Tiwari A, Pandey AC (2012) Utility and Potential Application of Nanomaterials in Medicine, in Biomedical Materials and Diagnostic Devices.

19. Singh RP, Choi JW, Tiwari A, Pandey AC (2014) Functional Nanomaterials for Multifarious Nanomedicine, in Biosensors Nanotechnology.

20. Ravindra PS, Choi JW (2010) Bio-nanomaterials for versatile bio-molecules detection technology. Letter to Editors. Advanced Materials Letters 1: 83-84.

21. Ravindra PS, Kang DY, Choi JW (2011) Nanofabrication of Bio-Self Assembled Monolayer and Its Electrochemical Property for Toxicant Detection. Journal of Nanoscience and Nanotechnology (JNN) Publisher: American Scientific Publishers 11: 408-412.

22. Ravindra PS, Avinash CP (2011) Silver Nano-Sieve using 1, 2-benzenedicarboxylic acid as a Sensor for Detecting Hydrogen Peroxide. Analytical Methods, Royal Society of Chemistry Publishing, Cambridge 3: 586-592.

23. Ravindra PS (2011) Biological approach of zinc oxide nanoparticles formation and its characterization. Advanced Materials Letters 2: 313-317.

24. Ravindra PS (2011) Silver/Polyaniline Nanocomposite for the electrocatalytic hydrazine oxidation. Ashutosh Tiwari and A C Pandey. Journal of Inorganic and Organometallic Polymers and Materials 21: 788-792.

25. Singh RP, Choi JW, Tiwari A, Pandey AC (2012) Biomimetic Materials Toward Application of Nanobiodevices, in Intelligent Nanomaterials: Processes, Properties, and Applications. John Wiley \& Sons Inc, Hoboken NJ USA 20 741-782.

26. Ahmed MU, Saaem I, Wu PC, Brown AS (2014) Personalized diagnostics and biosensors: a review of the biology and technology needed for personalized medicine. Crit Rev Biotechnol 34: 180-96.

27. Mahato K, Prasad A, Maurya PK, Chandra P (2016) Nanobiosensors: Nex Generation Point-of-Care Biomedical Devices for Personalized Diagnosis. J Anal Bioanal Tech 7: e125

28. Ravindra PS, Oh BK, Choi JW (2010) Application of peptide nucleic acid towards development of Nanobiosensor arrays. Bioelectrochemistry 79: 153-161.

29. Jeffrey NA, Paige WH, Olga L, Nilam CS, Jing Z, et al. (2008) Biosensing with plasmonic nanosensors. Nature Materials 7: 442-453.

30. Hsin NW, Andrew MFBS, Tuan Vo-Dinh (2015) Plasmonics-based SERS nanobiosensor for homogeneous. Nucleic acid detection 11: 811-814.

31. Vo Dinh T, Dhawan A, Norton SJ, Khoury CG, Wang HN, et al. (2010) Plasmonic nanoparticles and nanowires: design, fabrication and application in sensing. $J$ Phys Chem C 114: 7480-7488.

32. Pradyumna M, Sairam $Y$, Praharsha M, Neeharika K, Bishwambhar S, et al (2015) Flexible $\mathrm{Ag}-\mathrm{C}_{60}$ nano-biosensors based on surface plasmon coupled emission for clinical and forensic applications. Phys Chem Chem Phys 17 25049-25054

33. Gallegos D, Long K, Yu H, Clark P, Lin Y, et al. (2013) Label-free biodetection using a smartphone. Lab on a chip 13: 2124-2132.

34. Alexander S, La Jolla, Travis W, Venkatesh AG, Drew AH (2014) A low-cost smartphone-based electrochemical biosensor for point-of-care diagnostics. IEEE Biomedical Circuits and Systems Conference (BioCAS) Proceedings pp312-315.

35. Lillehoj PB, Huang MC, Truong N, Ho CM (2013) Rapid electrochemical detection on a mobile phone. Lab Chip 13: 2950-2955. 